\title{
Temperature dependence of the LO phonon sidebands in free exciton emission of GaN
}

\author{
S. J. Xu $\mathrm{u}^{\mathrm{a})}$ and G. Q. Li \\ Department of Physics and HKU-CAS Joint Laboratory on New Materials, The University of Hong Kong, \\ Pokfulam Road, Hong Kong, China \\ S.-J. Xiong \\ Department of Physics and HKU-CAS Joint Laboratory on New Materials, The University of Hong Kong, \\ Pokfulam Road, Hong Kong, China and National Laboratory of Solid State Microstructures \\ and Department of Physics, Nanjing University, Nanjing 210093, China \\ C. M. Che \\ Department of Chemistry and HKU-CAS Joint Laboratory on New Materials, The University of Hong \\ Kong, Pokfulam Road, Hong Kong, China
}

(Received 24 June 2005; accepted 13 February 2006; published online 5 April 2006)

\begin{abstract}
Temperature-dependent radiative recombination of free excitons involving one or two LO phonons in $\mathrm{GaN}$ is investigated in detail. It is found that both phonon sidebands possess asymmetric lineshapes and their energy spacings from the zero-phonon line strongly deviate from the characteristic energy of LO phonons as the temperature increases. Furthermore, the deviation rates of one- and two-phonon sidebands are significantly different. Segall-Mahan [Phys. Rev. 171, 935 (1968)] theory, taking the exciton-photon and exciton-phonon interactions into account, is employed to calculate the sidebands of one or two LO phonons for free excitons in a wide temperature range. Excellent agreement between the theory and experiment is achieved by using only one adjustable parameter, which leads to determination of the effective mass of heavy holes $\left(\sim 0.5 m_{0}\right)$. $\odot 2006$ American Institute of Physics. [DOI: 10.1063/1.2188034]
\end{abstract}

\section{INTRODUCTION}

Photon-exciton-phonon interactions are a fundamental many-body issue in solid-state materials, in particular, in direct wide gap semiconductors. ${ }^{1,2}$ Such interactions have been recognized to play an essential role in the intrinsic optical properties of these materials for a long time..$^{3-5}$ These references have provided the principal understanding of the photon-exciton-phonon coupling and its effect on absorption and reflectance of photon in nonmetallic materials. On the other hand, for photon emission, especially for phononassisted emission, our understanding is inadequate. ${ }^{1,6}$ A main reason for this is that such a photon emission or luminescence process involves the complicated interplay of different kinds of quasiparticles. Based on the Green's function method, Segall and Mahan ${ }^{7}$ quantum-mechanically treated the LO phonon-assisted luminescence of free excitons in semiconducting compounds with moderate to reasonably large band gaps. Under the weak coupling approximation, they derived the general expressions for one- and two-LOphonon-assisted luminescence spectra of free excitons. They also attempted to directly compare their theoretical results with available experimental luminescence spectra in $\mathrm{CdS}$ and $\mathrm{ZnO}$. In recent years, $\mathrm{GaN}$, which was earlier known to be a wide band gap semiconductor with the wurtzite structure, ${ }^{8,9}$ has been extensively investigated due to the first demonstration of the GaN-based bright light-emitting diode with short wavelength by Nakamura in $1993 .{ }^{10}$ However, the current status in the study of $\mathrm{GaN}$ is that the empirical technological

\footnotetext{
${ }^{a)}$ Electronic mail: sjxu@hkucc.hku.hk
}

development is advancing rapidly while a lot of fundamental issues are still far from complete understanding. ${ }^{11}$ This is particularly true for the LO phonon-assisted photon emission of free excitons in GaN. The present growth technology of high-quality GaN epilayers and even free-standing bulk has enabled efforts to revisit this issue in detail. ${ }^{12-18}$ Although these experimental studies have significantly enriched our knowledge about the photon-exciton-phonon interactions in $\mathrm{GaN}$, a firm understanding of the LO-phonon-assisted photon emission of free excitons is still lacking. Obviously, such an understanding is of considerable fundamental and technological importance.

In this paper, we experimentally and theoretically study the temperature-dependent LO-phonon-assisted luminescence of free excitons in GaN. A direct comparison between theory and experiment is made. All the aspects of both oneand two-LO-phonon-assisted luminescence spectra, such as linewidth, asymmetry, and peak shift are well interpreted in a broad temperature range.

\section{EXPERIMENT}

The high-quality GaN sample used in the present study was a $2.88 \mu \mathrm{m} \mathrm{GaN}$ epilayer grown on sapphire, followed by a $40 \mathrm{~nm} \mathrm{GaN}$ nucleation layer. The sample was prepared with a metalorganic vapor phase epitaxy. In the photoluminescence measurements, the samples were mounted on the cold finger of a Janis closed-cycle cryostat with varying temperature from 3.5 to $300 \mathrm{~K}$, and excited by the $325 \mathrm{~nm}$ line of a Kimmon $\mathrm{He}-\mathrm{Cd} \mathrm{cw}$ laser with output power of $40 \mathrm{~mW}$. The emission signal was dispersed by a SPEX 750M mono- 


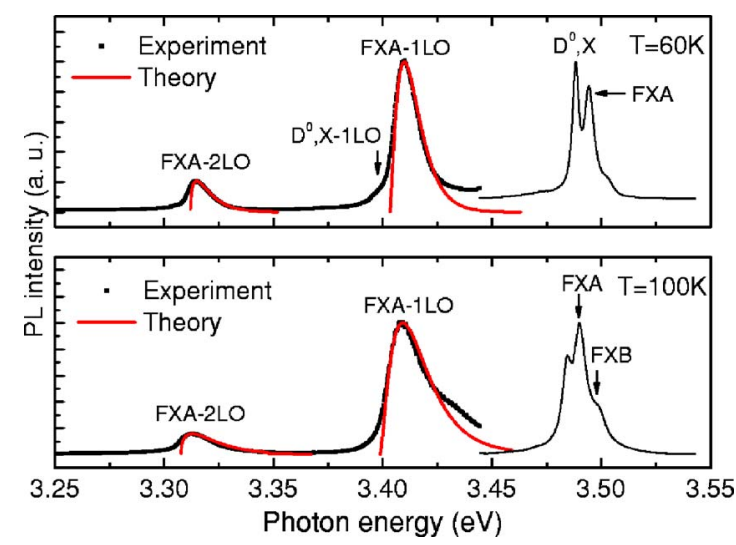

FIG. 1. (Color online) Two representative LO-phonon-assisted luminescence spectra (solid squares) at 60 and $100 \mathrm{~K}$. The corresponding zerophonon lines are also plotted as the thiner solid lines. The theoretical curves of the first-order and second-order LO phonon sidebands are given by the thicker solid lines.

chromator and detected with a Hamamatsu R928 photomultiplier. The details of the experimental apparatus have been previously described elsewhere. ${ }^{17}$

\section{EXPERIMENTAL RESULTS AND THEORETICAL CALCULATION}

As reported in literature, ${ }^{12,16}$ various excitonic resonant transitions (sometimes called zero-LO phonon lines) keep dominant in the PL spectra of GaN even at room temperature. For the sample studied in the work, $80 \mathrm{~K}$ is a dividing temperature below which the resonant line of the bound excitons dominates. When the temperature is higher than $80 \mathrm{~K}$ the resonant line of free excitons becomes dominant. ${ }^{17} \mathrm{We}$ are primarily concerned with the LO-phonon-assisted radiative decay of the free excitons in the present study. In such radiative decays of free excitons, the generation of photons accompanies with the emission of one or more LO phonons, leading to the so-called LO phonon sidebands. For GaN with the wurtzite structure, the characteristic energy of its $A_{1}-\mathrm{LO}$ vibration mode is as large as $91 \mathrm{meV}$. The LO phonon sidebands are thus well resolved from the zero-phonon lines. Due to different coupling strengths of the bound excitons and free excitons with LO phonons, the dividing temperature for the phonon sidebands is only about $25 \mathrm{~K},{ }^{17}$ i.e., the LO phonon sidebands of the free excitons are stronger than those of the bound excitons when the temperature is beyond $25 \mathrm{~K}$. Figure 1 shows two representative LO-phonon-assisted luminescence spectra (solid squares) at 60 and $100 \mathrm{~K}$. In the concerned spectral range, the one (denoted by FXA-1LO) and two-phonon sidebands (FXA-2LO) of the free exciton transition (FXA) can be clearly resolved. In order to provide a general picture of the exciton emissions, the corresponding zero-phonon lines are also shown in Fig. 1. In this spectral range, the neutral-donor-bound exciton (denoted by $D^{0}, X$ ), the free exciton A (marked by FXA, associated with $\Gamma_{9}$ valence band) and the free exciton B (FXB, associated with $\Gamma_{7}$ valence band) can be seen. At $60 \mathrm{~K}$, the remaining first-order LO-phonon sideband $\left(D^{0}, X-1 \mathrm{LO}\right)$ of the $D^{0}, X$ is still resolved. In the present work, we restrict our discussion on the first-order and second-order LO-phonon sidebands of the
FXA. The solid squares represent the measured spectra while the solid lines are the theoretical spectra obtained from the use of Segall-Mahan's model. ${ }^{7}$ It is noted that both peaks are asymmetric. Moreover, the asymmetry of the peaks increases as the temperature increases. Actually, as shown later, the increase rate of the asymmetry with temperature are apparently different between two peaks. We also found that the one-phonon peak is always broader than that of the twophonon peak in the moderately high temperature range where the two peaks are well defined. More interesting is that the energetic positions of the two peaks strongly blueshift with the temperature in different rate. These spectral features unambiguously show that the annihilation of free excitons accompanied with generation of one phonon essentially differs from that with generation of two phonons. For quantitative interpretation of these features, we employ the Segall-Mahan theory to calculate one- and two-LO-phononassisted luminescence spectra of free excitons.

The Hamiltonian for the photon-exciton-phonon coupling system can be written as ${ }^{7}$

$$
\begin{aligned}
H= & \sum_{\lambda K} \epsilon_{\lambda K} c_{\lambda K}^{\dagger} c_{\lambda K}+\sum_{q} \omega_{q} a_{q}^{\dagger} a_{q}+\sum_{K} \omega_{K} \alpha_{K}^{\dagger} \alpha_{K} \\
& +\sum_{\lambda K} \frac{M_{\lambda}(K)}{\sqrt{\omega_{K}}}\left(c_{\lambda K}^{\dagger} \alpha_{K}+\alpha_{K}^{\dagger} c_{\lambda K}\right) \\
& +\sum_{\lambda, \lambda^{\prime} ; K, q} V_{\lambda \lambda^{\prime}}(q) c_{\lambda, K+q}^{\dagger} c_{\lambda^{\prime} K}\left(a_{q}+a_{q}^{\dagger}\right),
\end{aligned}
$$

where $c_{\lambda K}^{\dagger}, a_{q}^{\dagger}$, and $\alpha_{K}^{\dagger}$ are the creation operators for exciton, phonon, and photon, $\epsilon_{\lambda K}, \omega_{q}$, and $\omega_{K}$ are energies of exciton, phonon, and photon, respectively, $M_{\lambda}$ is the exciton-photon matrix element, and $V_{\lambda \lambda^{\prime}}$ the coupling strength between exciton and phonon. The optical absorption coefficient can be evaluated from the change rate of photon number operator $N_{K}=\alpha_{K}^{\dagger} \alpha_{K}$,

$$
\partial N_{K} / \partial t=i\left[H, N_{K}\right]=i\left[H_{\mathrm{ex}-R}, N_{K}\right],
$$

where $H_{\mathrm{ex}-R}$ is the interaction term between excitons and photons [i.e., the forth term on the right hand of Eq. (1)]. The general expression of the absorption probability per photon for the photon-exciton-phonon coupling system can be derived by using the Green's function approach ${ }^{7}$

$$
\begin{aligned}
\omega W_{\mathrm{abs}}(\omega)= & \sum_{\lambda}\left|M_{\lambda}\right|^{2} A_{\lambda}(\omega)+2 \sum_{\lambda \neq \lambda^{\prime}} M_{\lambda} M_{\lambda^{\prime}} \\
& \times\left\{\operatorname{Re} \Sigma_{\lambda \lambda^{\prime}}(\omega) A_{\lambda}(\omega) \operatorname{Re} G_{\lambda^{\prime}}(\omega)-\operatorname{Im} \Sigma_{\lambda \lambda^{\prime}}(\omega)\right. \\
& \left.\times\left[\operatorname{Re} G_{\lambda}(\omega) \operatorname{Re} G_{\lambda^{\prime}}(\omega)-\frac{1}{4} A_{\lambda}(\omega) A_{\lambda^{\prime}}(\omega)\right]\right\},
\end{aligned}
$$

where $G_{\lambda}(\omega)$ is the Fourier transformation of the retarded Green's function defined as

$$
G_{\lambda \lambda^{\prime}}\left(K, t-t^{\prime}\right)=-i \theta\left(t-t^{\prime}\right)\left\langle\left[c_{\lambda K}(t), c_{\lambda^{\prime}, K}^{\dagger}\left(t^{\prime}\right)\right]\right\rangle,
$$

$\left(G_{\lambda}=G_{\lambda, \lambda}\right), A_{\lambda}(\omega)$ is the spectral density 


$$
A_{\lambda}(\omega)=-2 \operatorname{Im} G_{\lambda}(0, \omega),
$$

and $\Sigma_{\lambda, \lambda^{\prime}}(\omega) \equiv \Sigma_{\lambda, \lambda^{\prime}}(K=0, \omega)$ is the self-energy. Here we set $K=0$ for optical absorption.

As noted by Segall and Mahan, under the full thermal equilibrium and nondegenerate conditions (i.e., the exciton distribution can be well approximated by the Boltzmann distribution), the relationship between the transition probabilities of emission and absorption at the same frequency can be quantitatively expressed as ${ }^{7}$

$$
W_{e m}(\omega) \propto e^{-\omega / k_{B} T} W_{\mathrm{abs}}(\omega) .
$$

Therefore, one can find emission from Eq. (4) after getting the absorption. For the simultaneous absorption of a photon and $m$ LO phonons, the absorption coefficient $\alpha_{m}$ is given by $^{7}$

$$
\alpha_{m}(\omega) \propto W_{\mathrm{abs}}^{(m)}(\omega)=\left[N\left(\omega_{l}\right)\right]^{m}\left|M_{m}(\omega)\right|^{2},
$$

where

$$
N\left(\omega_{l}\right)=\frac{1}{e^{\omega_{l} / k_{B} T}-1} .
$$

The factor $\left|M_{m}\right|^{2}$ is the square of the overall matrix element.

According to the perturbation theory, the absorption coefficient for the process involving one phonon can be calculated by ${ }^{4,7}$

$$
\begin{aligned}
\alpha_{1}(\omega)= & \frac{4 \pi e^{2} \beta_{A, 1} \omega_{l}\left(\epsilon_{\infty}^{-1}-\epsilon_{s}^{-1}\right)}{4 a \hbar c B \sqrt{\epsilon^{\prime}}}\left(\frac{E_{A, 1}}{E_{A, 1}-\omega}\right)^{2} \\
& \times\left(\frac{M_{\|} B}{\mu_{\perp} \Delta}\right)^{1 / 2} N\left(\omega_{l}\right) I(\omega),
\end{aligned}
$$

where

$$
\begin{aligned}
I(\omega)= & \int_{0}^{-1} d x\left[1+\frac{M_{\|}-M_{\perp}}{M_{\perp}} x^{2}\right]^{-1} \\
& \times\left\{\frac{2\left[(1+\eta)^{2}-S_{e}^{2}\right]}{S_{e}\left[(1+\eta)^{2}+S_{e}^{2}\right]^{2}} \operatorname{Im} F_{e}(\eta)\right. \\
& \left.+\frac{4(1+\eta)}{\left[(1+\eta)^{2}+S_{e}^{2}\right]^{2}} \operatorname{Re} F_{e}(\eta)-[e \rightarrow h]\right\}^{2} .
\end{aligned}
$$

Here, $a$ and $B$ are the Bohr radius and the binding energy of excitons, $\epsilon_{\infty}$ and $\epsilon_{s}$ are dielectric constants at high and low frequencies, respectively, $4 \pi \beta_{A, 1}$ denotes the contribution of the $n=1 A$-exciton state to the polarizability, $E_{A, 1}$ is its energy, $M_{\|}$is the total masses of exciton in the $c$ axis, $\mu_{\perp}$ is the reduced mass in the direction perpendicular to the $c$ axis, $\epsilon^{\prime}$ is the dielectric constant at $E_{A 1}$ in absence of $n=1$ exciton, $\eta^{2}=\left(E_{G}-\omega\right) / B, \quad S_{e(h)}=\left[m_{h(e) \perp} / M_{\perp}\right] \mathbf{Q}^{[e(h)]} a \quad$ with $\quad \mathbf{Q}^{e(h)}$ $=\left\{q_{x}, q_{y}, q_{z}\left[m_{h(e) \|} M_{\perp} m_{e(h) \perp} / m_{h(e) \perp} M_{\|} m_{e(h) \|}\right]^{1 / 2}\right\}, \quad \Delta=\omega+\omega_{l}$ $-E_{A, 1}$, and

$$
\begin{aligned}
& F_{e}(\eta)={ }_{2} F_{1}\left(2,1-\eta^{-1}, 2-\eta^{-1}, \chi\right), \\
& \chi=\left[1-\eta^{2}+S_{e}^{2}-2 i S_{e} \eta\right] /\left[(1+\eta)^{2}+S_{e}^{2}\right],
\end{aligned}
$$

with ${ }_{2} F_{1}$ being the hypergeometric function. The absorption coefficient for the process involving two phonons is 4,7

$$
\begin{aligned}
\alpha_{2}(\omega)= & \frac{e^{2} \epsilon_{s} \beta_{A, 1} \omega_{l}^{2}\left(\epsilon_{\infty}^{-1}-\epsilon_{s}^{-1}\right)}{16 a \hbar c B^{2} \sqrt{\epsilon^{\prime}}}\left(\frac{E_{A, 1}}{E_{A, 1}-\omega}\right)^{2} \\
& \times\left(\frac{M}{\mu}\right)^{3}\left(\frac{\left\langle E_{A, n^{\prime}}\right\rangle-E_{A, 1}}{\left\langle E_{A, n^{\prime}}\right\rangle-\omega}\right)^{2} N^{2}\left(\omega_{l}\right) \sum_{n} I_{n}(\omega),
\end{aligned}
$$

where $M=m_{e}+m_{h}, \mu^{-1}=m_{e}^{-1}+m_{h}^{-1}, m_{e(h)}=\left[m_{e(h), \perp}^{2} m_{e(h), \|}\right]^{1 / 3}$, $\left\langle E_{A, n^{\prime}}\right\rangle \sim E_{A, 1}+5 B$ is an approximate average energy of exciton levels with $n^{\prime} \geqslant 2$, and

$$
\begin{aligned}
I_{n}(\omega)= & \int_{0}^{\infty} \frac{d z}{z} \int_{\left|\beta_{n}-z\right|}^{\beta_{n}+z} \frac{d z^{\prime}}{z^{\prime}} \mid\left\langle 1\left|U\left(2 z a^{-1}\right)\right| 1\right\rangle\left\langle 1\left|U\left(2 z^{\prime} a^{-1}\right)\right| n\right\rangle \\
& \times\left[\left(b+z^{2}\right)^{-1}-\left(d+z^{2}\right)^{-1}\right] \\
& +\left(d+z^{2}\right)^{-1}\left\langle 1\left|U\left(2 z a^{-1}\right) U\left(2 z^{\prime} a^{-1}\right)\right| n\right\rangle+\left.\left(z \rightarrow z^{\prime}\right)\right|^{2},
\end{aligned}
$$

with $b=\left(E_{A, 1}-\omega_{l}-\omega\right) M / 4 \mu B, \quad d=\left(\left\langle E_{A, n^{\prime \prime}}-\omega_{l}-\omega\right) M / 4 \mu B\right.$, $\beta_{n}=x_{n} a / 2, x_{n}^{2}=2 M \hbar^{-2}\left(\omega+2 \omega_{l}-E_{A, n}\right)$, and $E_{A, n^{\prime \prime}}$ being taken as infinity. Here, the matrix element is

$$
\begin{aligned}
\langle n|U| 1\rangle= & \left\{S_{1}\left(p_{h}\right)+n^{-2}\left[\frac{1}{4} S_{3}\left(p_{h}\right)-\frac{1}{12} S_{4}\left(p_{h}\right)\right]\right\} \\
& -\left\{p_{h} \rightarrow p_{e}\right\},
\end{aligned}
$$

where

$$
\begin{aligned}
S_{\kappa}(p)= & -p^{-1} 2^{\kappa+1}\left(1+p^{2}\right)^{-\kappa-1} \exp \left[-2 /\left(1+p^{2}\right)\right] \\
& \times\left\{\sin \left[2 p /\left(1+p^{2}\right)\right] \operatorname{Re}(1-i p)^{\kappa+1}\right. \\
& \left.+\cos \left[2 p /\left(1+p^{2}\right)\right] \operatorname{Im}(1-i p)^{\kappa+1}\right\},
\end{aligned}
$$

with $p_{e(h)}=\operatorname{qam}_{e(h)} / M$.

The theoretical spectra calculated using Eqs. (4), (6), and (7) are shown as the solid lines for direct comparison with the experimental spectra (solid squares) in Fig. 1. It can be seen that almost all spectral features including the position, the line shape, and the asymmetry of the measured sidebands are theoretically reproduced. This implies that the SegallMahan theory is applicable for calculating phonon-assisted luminescence spectra of free excitons in GaN. This coincidence between the theory and the experiment is furthermore illustrated by the temperature dependences of the linewidths of sidebands with one or two phonons extracted from the data and depicted in Fig. 2. Both the curves for the phonon sidebands with one and two phonons are in agreement with the experimental data. From the approximate expression for the free-exciton emission probability involving $m$ LO phonons ${ }^{7,19}$

$$
P_{m}(\omega) \sim \Delta_{m}^{(5 / 2)-m} e^{-\Delta_{m} / k_{B} T},
$$

with $\Delta_{m}=\omega+m \omega_{l}-E_{x 1}$, the linewidths can be approximately derived as $2.958 k_{B} T$ and $1.795 k_{B} T$ for the simultaneous emissions of one and two phonons, respectively. These approximate results are shown in Fig. 2 with light lines. Compared with the approximate results, the present theoretical calculation taking into account the intermediate excitonic states with $n>1$ gives an evident correction for $T>0$. For the one-phonon process this correction is negative from the sign of the matrix elements, while for the two-phonon pro- 


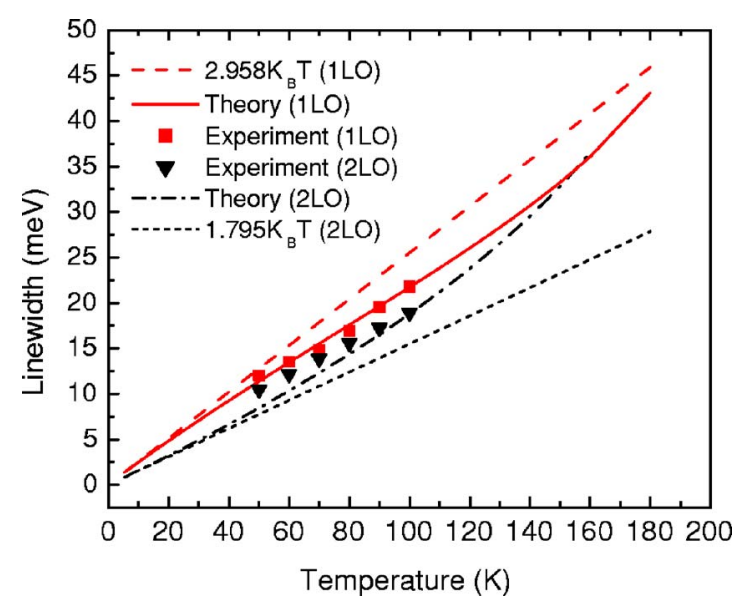

FIG. 2. (Color online) Linewidth of sidebands with one and two phonons as functions of temperature. The lines are the calculated results, and the filled symbols are the measured data.

cess it is positive due to the second-order terms in the perturbation theory.

Measured and theoretical temperature dependences of the energy spacings of the sidebands with one and two phonons from the zero-phonon line are shown in Fig. 3. It should be noted that in order to clearly show departure of the energy spacings from a standard LO-phonon characteristic energy with increasing the temperature, half of the energy spacing between the second-order LO-phonon sideband and the zero-phonon line is plotted in Fig. 3. An excellent agreement between the theory and experiment is also achieved. Both the energy spacings generally decrease with increasing temperature in spite of the fact that they have different decrease rates. Such temperature dependences of the energy spacings between the LO phonon sidebands and their common principal line (the zero-phonon line) lead to significant deviation of the energy spacings from the integer folds of LO-phonon characteristic energy, in particular, at higher temperatures. Probably influenced by the great success of previous polaron models, the energy spacings between the phonon sidebands and the corresponding zero-phonon line have been simply thought to be always equal to the energies of phonons involved for a quite long time. For example, see a recent

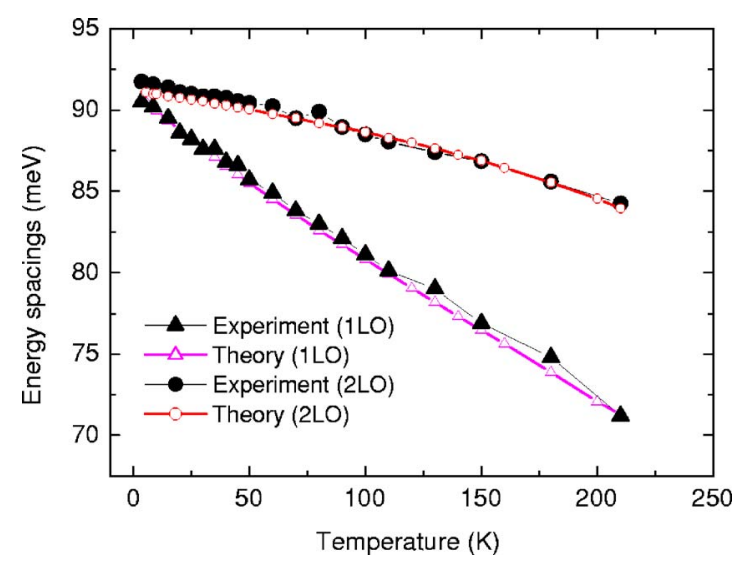

FIG. 3. (Color online) Temperature dependence of the energy spacing from the sidebands with one and two phonons to the zero-phonon line obtained from the theory and form the experiment. review article. ${ }^{18}$ Presently, our experimental results and rigorous theoretical calculation unambiguously show that the energy spacings between the LO phonon sidebands and the zero phone line of free excitons are temperature dependent. As stated earlier, the Segall-Mahan model employed in the present theoretical calculation takes the contributions of the intermediate excitonic states into account. This leads to not only inherent asymmetric line shapes of the phonon sidebands but also negative corrections to the previous approximate values of the energy spacings for both the one-phonon and two-phonon processes. These corrections are actually originated from the renormalization of the energy levels due to the phonon scattering associated with the intermediate excitonic states. ${ }^{1,7}$ It can be seen that the correction to the onephonon process is larger than that of the two-phonon process, implying the perturbation nature of the scattering in which the correction is decaying with the order. For both cases the correction increases with increasing the temperature, because at higher temperatures more intermediate excitonic states are involved.

In the calculations we use the following parameters for GaN. ${ }^{10,17,20,21} \quad m_{e}=0.2 m_{0}, \quad m_{h \perp}=0.5 m_{0}, \quad m_{h \|}=0.6 m_{0}, \quad B$ $=0.0254 \mathrm{eV}, \quad \omega_{l}=0.0912 \mathrm{eV}, \quad \epsilon_{s}=8.9, \quad \epsilon_{\infty}=5.35, \quad E_{A, 1}(T$ $=60 \mathrm{~K})=3.4944 \mathrm{eV}, \quad E_{A, 1}(T=100 \mathrm{~K})=3.4900 \mathrm{eV}, \quad$ and $4 \pi \beta_{A, 1}=0.0066$. It should be emphasized that all the parameters, except the hole effective mass, are taken from those of the material. The best agreement is achieved between the theory and the experiment when we choose $m_{h \perp}=0.5 m_{0}$, $m_{h \|}=0.6 m_{0}$, which are much lower than the results of band calculations, $m_{h \perp} \sim m_{h \|} \sim 1.5 m_{0}$. From the excellent agreement between the theory and the experiment, we suggest that the values used here are more adequate. This is supported from the experimental value of the binding energy. The relation between the binding energy and the reduced mass of an exciton is ${ }^{22}$

$$
B=\left(\frac{\mu}{m_{0} \epsilon_{s}^{2}}\right) \mathrm{Ry},
$$

with $\mathrm{Ry}=13.6 \mathrm{eV}$. From this relation and measured values of $B$ and $\epsilon_{s}$ listed above, the reduced mass is $\mu=0.148 m_{0}$. Since the effective mass of electron has been well measured as $m_{e}=0.2 m_{0}$, from $\mu$ one can determine the effective mass of hole as $m_{h} \sim 0.57 m_{0}$. This supports the values of $m_{h \perp}$ and $m_{h \|}$ used here for giving the best consistence of the theory and the experiment. Thus, the present measurement of the temperature-dependent photon emission spectra provides another way of determining the hole effective mass in GaN, and the obtained result coincides the relation between the binding energy and the reduced mass, although it is different from the results of the band calculations.

\section{CONCLUSION}

As a summary, we experimentally and theoretically investigate the temperature-dependent radiative recombination of free excitons involving one or two LO phonons in GaN. It is found that both phonon sidebands possess asymmetric line shape and different temperature dependence. In a wide temperature range, the calculated spectra from the Segall-Mahan 
theory are in excellent agreement with the measured results. This study provides a method of determining the effective mass of holes in GaN.

\section{ACKNOWLEDGMENTS}

The work in Hong Kong was supported by the HK-RGC CERG Grant No. HKU 7036/03P. S-JX gratefully acknowledges research support from Chinese National Foundation of Nature Sciences (Grant Nos. 60276005 and 10474033). One of the authors (S.J.X.) wishes to thank Dr. W. Liu and Professor M.F. Li for providing GaN sample.

${ }^{1}$ Y. Toyozawa, Optical Processes in Solids (Cambridge University Press, Cambridge, 2003).

${ }^{2}$ E. I. Rashba and M. D. Sturge, Excitons (North-Holland, Amsterdam, 1982).

${ }^{3}$ J. J. Hopfield, Phys. Rev. 112, 1555 (1958); J. J. Hopfield, P. J. Dean, and D. G. Thomas, ibid. 158, 748 (1967).

${ }^{4}$ B. Segall, Phys. Rev. 150, 734 (1966); 163, 769 (1967).

${ }^{5}$ W. C. Tait and R. L. Weiher, Phys. Rev. 166, 769 (1968).

${ }^{6}$ For example, S. Permogorov, in Excitons, edited by E. I. Rashba and M. D. Sturge (North-Holland, Amsterdam, 1982), Chap. 5, p. 177.

${ }^{7}$ B. Segall and G. D. Mahan, Phys. Rev. 171, 935 (1968).

${ }^{8}$ R. Dingle, D. D. Sell, S. E. Stokowski, and M. Ilegems, Phys. Rev. B 4, 1211 (1971).
${ }^{9}$ B. Monemar, Phys. Rev. B 10, 676 (1974)

${ }^{10}$ S. Nakamura, S. Pearton, and G. Fasol, The Blue Laser Diode, 2nd ed. (Springer, Berlin, 2000).

${ }^{11}$ N. M. Johnson, A. V. Numrikko, and S. P. DenBaars, Phys. Today 53, 31 (2000).

${ }^{12}$ D. Kovalev, B. Averboukh, D. Volm, B. K. Meyer, H. Amano, and I. Akasaki, Phys. Rev. B 54, 2518 (1996).

${ }^{13}$ I. A. Buyanova, J. P. Bergman, B. Monemar, H. Amano, and I. Akasaki, Mater. Sci. Eng., B 50, 130 (1997).

${ }^{14}$ W. Liu, M. F. Li, S. J. Xu, K. Uchida, and K. Matsumoto, Semicond. Sci. Technol. 13, 769 (1998).

${ }^{15}$ D. C. Reynolds, D. C. Look, B. Jogai, and R. J. Molnar, Solid State Commun. 108, 49 (1998).

${ }^{16}$ M. Leroux, N. Grandjean, B. Beaumont, G. Nataf, F. Semond, J. Massies, and P. Gibart, J. Appl. Phys. 86, 3721 (1999).

${ }^{17}$ S. J. Xu, W. Liu, and M. F. Li, Appl. Phys. Lett. 77, 3376 (2000); 81, 2959 (2002)

${ }^{18}$ X. B. Zhang, T. Taliercio, and S. Lefebvre, J. Phys.: Condens. Matter 13, 7053 (2001).

${ }^{19}$ E. Gross, S. Permogorov, and B. Razbirin, J. Phys. Chem. Solids 27, 1647 (1966).

${ }^{20}$ V. Bougrov, M. E. Levinshtein, S. L. Rumyantsev, A. Zubrilov, in Properties of Advanced Semiconductor Materials GaN, AlN, InN, BN, SiC, SiGe, edited by M. E. Levinshtein, S. L. Rumyantsev, and M. S. Shur (Wiley, New York, 2001), pp. 1-30.

${ }^{21}$ Y. C. Yeo, T. C. Chong, and M. F. Li, J. Appl. Phys. 83, 1429 (1998).

${ }^{22}$ P. Y. Yu and M. Cardona, Fundamentals of Semiconductors, 2nd ed. (Springer-Verlag, Berlin, 1999), p. 271. 